\title{
BMJ Open Effects of two types of smartphone- based stress management programmes on depressive and anxiety symptoms among hospital nurses in Vietnam: a protocol for three-arm randomised controlled trial
}

Kotaro Imamura, ${ }^{1}$ Thuy Thi Thu Tran, ${ }^{2}$ Huong Thanh Nguyen, ${ }^{3}$ Kazuto Kuribayashi, ${ }^{4}$ Asuka Sakuraya, ${ }^{1}$ Anh Quoc Nguyen, ${ }^{5}$ Thu Minh Bui, ${ }^{6}$ Quynh Thuy Nguyen, ${ }^{2}$ Kien Trung Nguyen, ${ }^{3}$ Giang Thi Huong Nguyen, ${ }^{6}$ Xuyen Thi Ngoc Tran, ${ }^{6}$ Tien Quang Truong, ${ }^{3}$ Melvyn W B Zhang, ${ }^{7}$ Harry Minas, ${ }^{8}$ Yuki Sekiya, ${ }^{1}$ Natsu Sasaki, ${ }^{1}$ Akizumi Tsutsumi, ${ }^{9}$ Norito Kawakami ${ }^{1}$

To cite: Imamura K, Tran TTT, Nguyen HT, et al. Effects of two types of smartphonebased stress management programmes on depressive and anxiety symptoms among hospital nurses in Vietnam: a protocol for three-arm randomised controlled trial. BMJ Open 2019;9:e25138. doi:10.1136/ bmjopen-2018-025138

- Prepublication history for this paper is available online To view these files, please visit the journal online (http://dx.doi. org/10.1136/bmjopen-2018025138).

Received 1 July 2018 Revised 7 January 2019 Accepted 26 February 2019

Check for updates

(C) Author(s) (or their employer(s)) 2019. Re-use permitted under CC BY-NC. No commercial re-use. See rights and permissions. Published by BMJ.

For numbered affiliations see end of article.

Correspondence to Professor Norito Kawakami; nkawakami@m.u-tokyo.ac.jp

\section{ABSTRACT}

Introduction Due to an increasing demand for healthcare in low-income and middle-income countries in Asia, it is important to develop a strategy to manage workrelated stress in healthcare settings, particularly among nurses in these countries. The purpose of this three-arm randomised controlled trial (RCT) is to examine the effects of a newly developed smartphone-based multimodule stress management programme on reducing severity of depressive and anxiety symptoms as primary outcomes at 3-month and 7-month follow-ups among hospital nurses in Vietnam.

Methods and analysis The target study population will be registered nurses working in a large general hospital (which employs approximately about 2000 nurses) in Vietnam. They will be invited to participate in this study. Participants who fulfil the eligibility criteria will be randomly allocated to the free-choice, multimodule stress management (intervention group $A, n=360$ ), the internet cognitive behavioural therapy (iCBT), that is, fixed-order stress management (intervention group $B, n=360$ ), or a treatment as usual control group $(n=360)$. Two types (free-choice and fixed sequential order) of smartphonebased six-module stress management programmes will be developed. Participants in the intervention groups will be required to complete one of the programmes within 10 weeks after the baseline survey. The primary outcomes are depressive and anxiety symptoms, measured by using the Depression Anxiety and Stress Scales (DASS) at 3-month and 7 month follow-ups.

Ethics and dissemination The study procedures have been approved by the Research Ethics Review Board of Graduate School of Medicine/Faculty of Medicine, the University of Tokyo (no 11991) and the Ethical Review Board for Biomedical Research of Hanoi University of Public Health (no 346/2018/YTCC-HD3). If a significant effect of the intervention programmes will be found in the $\mathrm{RCT}$, the programmes will be made available to all nurses
Strengths and limitations of this study

This will be the first randomised controlled trial to test the effectiveness of fully automated smartphone-based stress management programmes on improving depressive and anxiety symptoms and work-related outcomes among nurses in Vietnam.

- This study also intends to add evidence for the effect of e-stress management programmes on positive health and work-related outcomes (eg, work engagement and work performance) among nurses.

- A limitation of this study is that all outcomes will be measured by self-report, which may be affected by the perceptions or situational factors at work of the participants.

in the hospital including the control group. If the positive effects are found in this RCT, the e-stress management programmes will be disseminated to all nurses in Vietnam. Trial registration number UMIN000033139; Pre-results.

\section{INTRODUCTION}

Nurses can suffer from various work-related stresses related to factors such as workload, leadership/management style, professional conflict, emotional cost of caring, lack of reward and shift work. ${ }^{1}$ In addition, lack of stress management skills and/or organisational factors at work may contribute to difficulty in coping with stress. ${ }^{23}$ This often leads to severe psychological distress (eg, depression and anxiety), ${ }^{4}$ burnout, ${ }^{5}$ other health problems ${ }^{67}$ and deterioration in quality of life (QOL) and service provision. ${ }^{8}$ A shortage 
of nurses, population ageing and demands from service users (ie, patients and families) have increased pressure and stress on nurses and other healthcare professionals. ${ }^{9-11}$ Previous reports showed that deficits in the number of health service providers are very large in low-income and middle-income countries in Southeast Asia, ${ }^{12} 13$ where, despite the rapidly increasing quantitative and qualitative demands to medical care in the rapidly ageing society, there is a severe shortage of nurses, and many nurses lack the clinical skills to adequately respond to healthcare demands. ${ }^{13}$ Work-related stress has been increasing among nurses in Vietnam ${ }^{14}$ and other Southeast Asian countries. ${ }^{15-18}$ Moreover, work stress could also affect the quality of healthcare service in these countries. ${ }^{19}$ It is important to manage work-related stress in healthcare settings, particularly among nurses, focusing on Southeast Asian countries such as Vietnam.

For the working population, stress management based on cognitive behavioural therapy (CBT) has been shown to reduce depression/anxiety symptoms among workers. ${ }^{20}$ A recent meta-analysis reported that programmes combining CBT and coping flexibility showed the highest effect size ( $\mathrm{d}=1.45$ at 4 months follow-up) in the workplace. $^{21}$ Other meta-analyses showed a similar effect of programmes using CBT and relaxation on improving work-related stress among workers. ${ }^{22}{ }^{23}$ In the healthcare worker setting, CBT interventions have been shown to be effective. For instance, CBT, either with or without relaxation, showed a significant improving effect on stress symptoms among healthcare workers (standardised mean difference $[\mathrm{SMD}]=-0.38$ ) and especially among nurses $(\mathrm{SMD}=-0.34)$ at 6 -month follow-up. ${ }^{2}$ Computerised CBT delivered via the internet (iCBT) and other web-based interventions including cognitive behavioural techniques holds promise as a cost-effective method to make CBT accessible to individual workers. ${ }^{24}$ These iCBT programmes provide basic information and skills on the basis of CBT principles as face-to-face CBT programmes do, sometimes with a structured format that comprises educational lessons, homework assignments and supplementary resources. A recent literature review stated that the benefits of web-based intervention in the workplace include fewer constraints with regard to time and location, the potential to access a larger target group and protection of participant privacy-thereby reducing possible stigma with regard to seeking help for stress. ${ }^{24}$ Internet CBT interventions showed a small-to-moderate effect on increasing psychological well-being including reduction of psychological distress $(\mathrm{g}=0.37)$ and effective work outcomes such as engagement and productivity $(\mathrm{g}=0.25)$ at post intervention period, compared with the control condition. ${ }^{25}$ Another meta-analysis that examined the intervention effect at post intervention period and follow-up period (around 5 months) showed that eHealth interventions had a significant effect on improving mental health condition at both post intervention $(\mathrm{g}=0.24)$ and follow-up ( $\mathrm{g}=0.23)$ among employees. ${ }^{26}$ In addition, one randomised controlled trial (RCT) reported that web-based stress management programmes including cognitive behavioural techniques reduced perceived work-related stress among nurses in the USA. ${ }^{27}$ Internet CBT interventions might be effective in reducing work-related stress among nurses in low-income and middle-income countries. However, evidence obtained for other sector workers in high-income countries was not extended to nurses in low-income and middle-income countries in Asia. It is necessary to develop low-cost iCBT interventions for improving work stress and promoting mental health of nurses in low-income and middle-income countries and test its effectiveness.

An important challenge in applying web-based interventions is low adherence. Previous systematic reviews reported that completion of web-based psychological intervention programmes in the workplace was approximately $40 \%$, while the adherence rates varied considerably. ${ }^{25} \mathrm{~A}$ useful strategy to promote adherence to eHealth interventions is incorporating tailoring. ${ }^{28}$ For instance, a previous RCT showed that the attrition and adherence rate of an individually tailored iCBT programme appeared favourable compared with studies that applied non-tailored iCBT programmes. ${ }^{29}$ Even programmes that are not fully tailored but that allow participants to choose a module from multiple options based on their preference may be useful, while a typical iCBT programme provides multiple modules in a fixed sequential order. A comparison of the effects of these different types of iCBT programmes on adherence and outcomes has not been well studied before. Comparative effectiveness of these types of iCBT programmes may depend on the culture of the target country and the target population. The other approach that has become popular is to use smartphones as a medium for iCBT. Many smartphone apps for stress management use evidence-based strategies. ${ }^{30}$ However, only a small number of such programmes have been evaluated with an RCT, with very limited evidence in the working population. ${ }^{31}$

\section{Objectives}

Two types of smartphone-based multimodule stress management intervention programmes for reduction in symptoms of depression and anxiety with a similar number of modules and content will be developed for hospital nurses in Vietnam. The objectives of this study, using a three-arm RCT design, are as follows:

- To examine the effects of these intervention programmes on improving depressive and anxiety symptoms as primary outcomes, and on work engagement, work performance, stress symptoms, psychosocial work environment and health-related QOL as secondary outcomes at 3-month and 7-month follow-ups.

- To examine whether a free-choice sequence (ie, partially tailored) programme results in better adherence than completion of similar modules in a fixed sequential order. 
We chose Vietnam as a target country because of the degree of resources and challenges, improved mobile access to the internet, the increasing demands on medical services due to a rapidly ageing population and an increasing number of cases of burnout among nurses. The hypotheses of this study are as follows:

H1: The newly developed smartphone-based multimodule stress management intervention programmes will significantly improve the primary outcomes (ie, depressive and anxiety symptoms) among participants in the intervention groups compared with participants in the control group.

H2: The newly developed smartphone-based multimodule stress management intervention programmes will significantly improve the secondary outcomes (ie, work engagement, work performance, stress symptoms, psychosocial work environment and health-related QOL) among participants in the intervention groups compared with participants in the control group.

H3: Participants will show significantly better adherence (ie, completion rate of the programme) to the free-choice programme than to the fixed-sequence programme.

\section{METHODS AND ANALYSIS \\ Trial design}

The study will be a three-arm (including two different intervention groups), parallel-group, treatment as usual (TAU)-controlled, non-blinded randomised study. The allocation ratio of the intervention groups to the control group is 1:1:1. Participants will be recruited from a large general hospital in Hanoi, Vietnam, and randomly allocated to one of the three groups after they have completed a baseline questionnaire survey. Follow-up surveys will be conducted 3 and 7 months after the baseline. This protocol manuscript is written in accordance with the Standard Protocol Items: Recommendations for Interventional Trials guideline checklist. ${ }^{32}$

\section{Participants}

The target population of this RCT will be registered nurses working in a large general hospital (which employs approximately 2000 nurses) in Vietnam who will be invited to participate and selected according to the following criteria:

\section{Inclusion criteria}

1. Currently employed full time as a registered nurse.

2. Can access the internet via a mobile device such as a smartphone.

\section{Exclusion criteria}

1. Plan to change or quit the job in the next 7 months.

2. Assistant nurses and helpers.

3. Non-regular or part-time employed.

4. Sick leave for 15 or more days for a physical or mental condition in the past 3 months.
5. Current treatment for a mental health problem from a mental health professional.

\section{Procedure}

Figure 1 shows the participant flowchart of this trial. Our preliminary research reported that about $60 \%$ of the nurses in the hospital have their own smartphone. In addition, a previous RCT reported that about $10 \%$ of participants had to be excluded according to two exclusion criteria, which were having sick leave for 15 or more days for own health problems in the past 3 months and receiving medical treatment for a mental health problem during the past month. ${ }^{33}$

For this study, the clinical research coordinator (CRC) will send invitations to 2000 nurses, of whom 1200 are expected to have their own smartphone and give informed consent, and 1080 are expected to be eligible. These 1080 will be randomised to either one intervention group $(n=360)$, the other intervention group $(n=360)$, or the control group $(n=360)$. Participants in the two intervention groups will be required to complete the intervention programmes within 10 weeks after the baseline survey.

An invitation letter to all nurses will include a full explanation of the study and information on the eligibility criteria. After reading the explanation of the study, potential participants will be invited to give their written consent to participate in the study, and to complete and return the baseline survey to CRC within a week.

\section{Intervention programmes}

In this study, two smartphone-based six-module stress management programmes will be used. One (programme A) is a free-choice, multimodule stress management programme in which respondents are allowed to select one module per week in any order they prefer. The other (programme B) is a fixed-sequence, multimodule stress management programme in which respondents are required to study modules in a fixed order, one module per week. For both programmes, it will take about $15 \mathrm{~min}$ to complete each module.

Programme A includes six modules that provide six evidence-based stress management skills. This programme is based on a previous web-based stress management programme aimed to improve psychological distress of office workers, ${ }^{34}$ and modified on the basis of intensive consultation with nurses in Vietnam, for example, replacing one module (on physical activity for stress management) with another (self-compassion). Participants may choose one module per week in any order they prefer. The programme includes behavioural activation (module 1$)$, cognitive restructuring (module 2 ), problem solving (module 3 ), assertiveness (module 4), self-compassion (module 5) and job crafting (module 6).

Programme B also includes six modules that provide CBT-based stress management skills, developed based on a previous iCBT programme that successfully improved depression in office workers. ${ }^{33}$ The six modules are 
Baseline survey (T1)

Participants will be recruited from a general hospital (about 2,000) in Vietnam

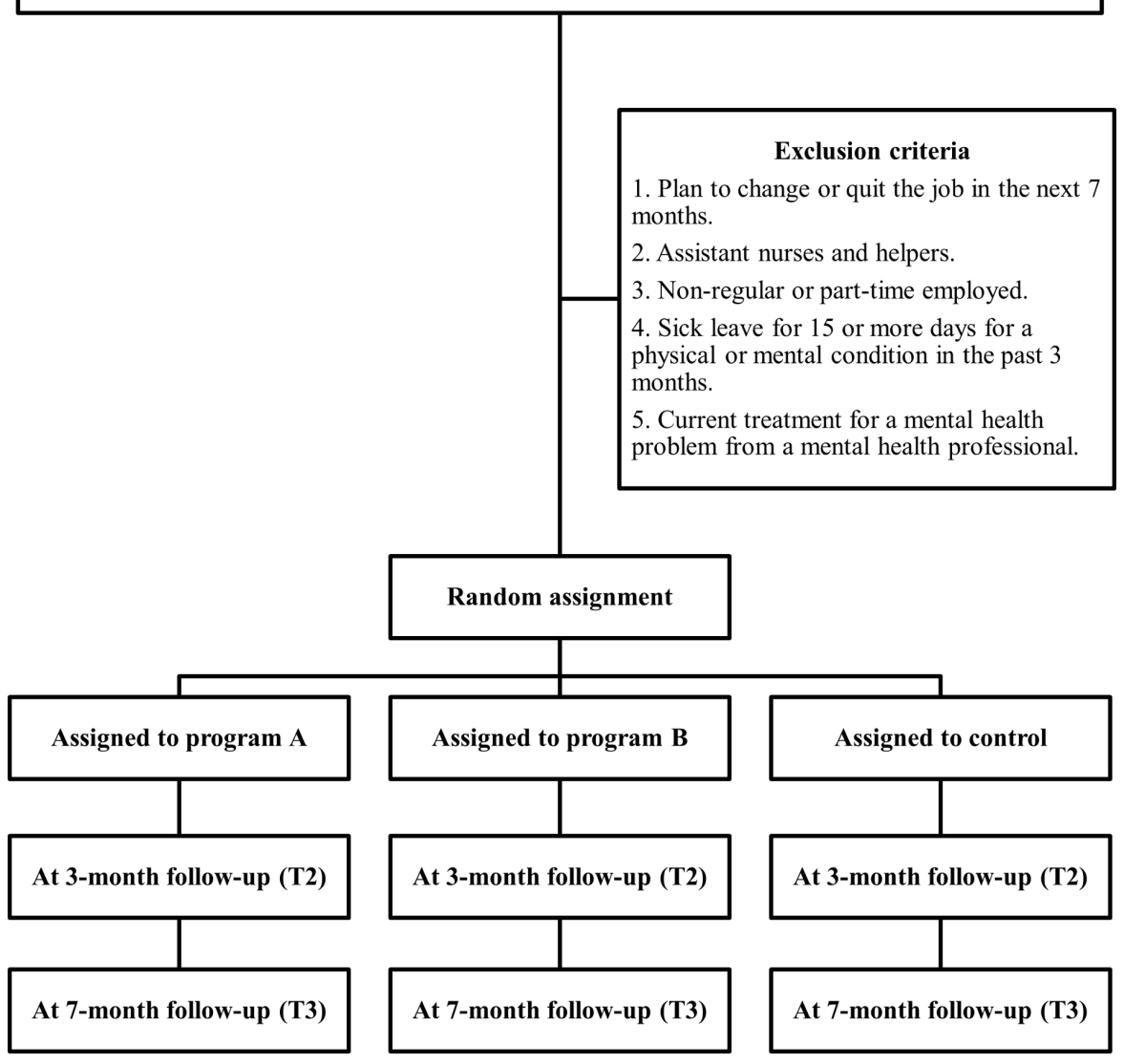

Figure 1 Participant flow chart.

presented in a fixed order, with one module accessible per week, from module 1 to module 6 . The programme has two already established CBT packages as its basis. One is the cognitive therapy programme developed by Beck. ${ }^{35}$ The other is the 'Coping with Depression' programme developed by Lewinsohn. ${ }^{36}$ The programme includes transactional model of stress and coping (module 1), selfcase formulation based on cognitive behavioural model (module 2), behavioural activation skills (module 3), cognitive restructuring skills (modules 4 and 5), problem-solving skills (module 6 ) and relaxation skills (module 5). The programme is modified so that the content is relevant to and appropriate for the working situation and work culture of nurses in Vietnam. For instance, case stories are modified reflecting major stressors (ie, job overload) of these nurses.

Table 1 shows the stress management techniques included in programmes A and B. Behavioural activation, cognitive restructuring and problem-solving techniques are included in both programmes A and B. Assertiveness, self-compassion and job crafting techniques are included only in programme A. The transactional model of stress and coping, self-case formulation based on cognitive behavioural model and relaxation techniques are included only in programme B. Details of each of the components are as follows.
Transactional model of stress and coping (module 1 in programme B)

Transactional stress model is defined as perceptions that demands exceed coping strategies. ${ }^{37}$ According to this model, an individual's reaction to stressors is determined, in part, by their own appraisal of the stressor. In keeping with this model, stress will be defined as the psychological

Table 1 Contents of the free-choice (programme A) and fixed-order (programme B) stress management programmes

Techniques for stress Programme A Programme B management (Module No) (Module No)

\begin{tabular}{|c|c|c|}
\hline $\begin{array}{l}\text { Transactional model of } \\
\text { stress and coping }\end{array}$ & Not included & Module 1 \\
\hline $\begin{array}{l}\text { Self-case formulation } \\
\text { based on cognitive } \\
\text { behavioural model }\end{array}$ & Not included & Module 2 \\
\hline Behavioural activation & Module 1 & Module 3 \\
\hline Cognitive restructuring & Module 2 & Modules 4 and 5 \\
\hline Relaxation & Not included & Module 5 \\
\hline Problem solving & Module 3 & Module 6 \\
\hline Assertiveness & Module 4 & Not included \\
\hline Self-compassion & Module 5 & Not included \\
\hline Job crafting & Module 6 & Not included \\
\hline
\end{tabular}


response to a situation or stimuli whereby an individual appraises the situation or stressor as exceeding their capabilities or resources. In this module, participants learn about the relationship between stressors and stress reactions.

\section{Self-case formulation based on cognitive behavioural model (module 2 in programme B)}

In this module, participants learn about a cognitive behavioural (CB) model, especially the five-part model (situation, thoughts, emotions, behaviour and physical feelings $)^{38}$ and a self-case formulation based on this model. Case formulation is a method used to understand the problem of a client. ${ }^{39}$ Case formulation is necessary for clients to choose an appropriate approach to change the vicious circles of these five areas.

Behavioural activation (module 1 in programme $A$ and module 3 in programme B)

Behavioural activation, one of the most readily applied techniques in CBT, is a process to increase pleasurable and rewarding activities using behavioural strategies such as activity scheduling. ${ }^{40}$ This module/programme provides a behavioural activation technique on enhancing participants' liveliness. Participants learn about a theory of behavioural activation and how to plan an activity schedule for increasing pleasant activities.

\section{Cognitive restructuring (module 2 in programme A and modules 4}

and 5 in programme B)

The cognitive restructuring technique is one of the standard cognitive approaches of CBT utilised to change an automatic negative thought into an actual, realistic and flexible thought. ${ }^{35}$ This module gives a lecture on a cognitive ABC model (Activating/Actual event, Belief and Consequence $)^{35} 4142$ and on identifying the automatic thoughts that cause a negative mood. Participants learn cognitive restructuring skills to change an automatic negative thought into an actual thought.

\section{Relaxation (module 5 in programme B)}

Relaxation techniques are often added to the CBT intervention for workers, and they have shown significant effects on improving depression. ${ }^{22}$ In the latter half of the module 5 , participants learn a relaxation technique using a breathing method.

\section{Problem solving (module 3 in programme A and module 6 in programme B)}

Problem-solving technique is a $\mathrm{CB}$ intervention that focuses on training adaptive problem-solving attitudes and skills. ${ }^{43}$ A rational problem-solving style involves the deliberate and systematic application of four major problem-solving skills: (1) problem definition and formulation, (2) generation of alternative solutions, (3) decision making and (4) solution implementation and verification. ${ }^{44}$ In this module, participants learn problem-solving skills to sort out the problem and make a list of solutions.
Assertiveness (module 4 in programme A)

Assertiveness is typically defined as the legitimate and honest expression of one's personal rights, feelings, beliefs and interests without violating or denying the rights of others. ${ }^{45} 46$ In order to communicate assertively, the Describe, Express, Specify, and Choose or Consequence (DESC) script is used. ${ }^{47}$ In this module, participants learn assertiveness skills to appropriately communicate their concerns to supervisors, coworkers or subordinates, based on the DESC script.

\section{Self-compassion (module 5 in programme A)}

Self-compassion describes a positive and caring attitude of a person towards herself or himself in the face of failures and individual shortcomings. ${ }^{48}$ As a result of this caring attitude, individuals high in self-compassion are assumed to experience higher individual well-being. There are three interrelated elements that determine the self-compassionate reactions to negative events and experiences: self-kindness, sense of common humanity and mindfulness. ${ }^{48}$ In this module, participants learn a concept of self-compassion and how to express compassion towards themselves.

\section{Job crafting (module 6 in programme A)}

Job crafting is defined as the physical and cognitive change individuals make in the task or relational boundaries of their work ${ }^{, 50}$ and consists of the following three components: changing the job's boundaries (task crafting), changing the relational boundaries (relational crafting) and changing the cognitive task boundaries (cognitive crafting) ${ }^{50}$ In this module, participants learn about the concept of job crafting and how to craft their own job.

\section{Intervention groups}

Participants in the intervention groups will be required to complete programme A or B within 10 weeks after the baseline survey. Participants will be provided with their own ID and password to sign in to the programme and asked not to tell anyone else this information. The participants will be reminded by email to complete the programme if they have not already done so. Reminders will be sent from the research office. Before the start of the intervention programme, participants in the intervention groups download the apps and view an introduction module that provides general explanations of the programmes. The introduction module is common for the two intervention programmes with brief explanations about the psychological stress model, self-assessment of their own mood using Kessler's Psychological Distress Scale (K6) ${ }^{51}$ and an introduction of the two intervention programmes. Participants can contact CRC if they have trouble downloading or using the apps. After 3 months from baseline survey, the intervention programmes will be closed by CRC.

\section{Control group}

Participants in the control group do not receive any intervention programmes during the intervention and 
Table 2 Overview of outcome measurements

\begin{tabular}{|c|c|c|c|c|}
\hline Measurement & Aim & $\begin{array}{l}\text { Baseline } \\
\text { (T1) }\end{array}$ & $\begin{array}{l}3 M F / U \\
\text { (T2) }\end{array}$ & $\begin{array}{l}7 \mathrm{M} F / U \\
\text { (T3) }\end{array}$ \\
\hline \multicolumn{5}{|c|}{ Primary outcomes } \\
\hline DASS & Severity of anxiety symptoms & $x$ & $x$ & $x$ \\
\hline \multicolumn{5}{|c|}{ Secondary outcomes } \\
\hline DASS & Severity of stress symptoms & $x$ & $x$ & $x$ \\
\hline JCQ & Psychosocial work environment & $x$ & $x$ & $x$ \\
\hline$E Q-5 D$ & Health-related quality of life & $x$ & $x$ & $x$ \\
\hline
\end{tabular}

DASS, Depression Anxiety and Stress Scales; HPQ, Health and Work Performance Questionnaire; JCQ, Job Content Questionnaire; UWES, Utrecht Work Engagement Scale.

follow-up period ( 7 months). Participants both in the intervention group and the control group will be able to use an internal employee assistance programme service as a TAU. Participants in the control group will be provided the opportunity to use the intervention programmes after the 7-month follow-up.

\section{Outcomes}

Table 2 shows an overview of the outcome measures. All outcome measures will be assessed at baseline, 3-month (the end of the intervention period) and 7-month follow-ups. Non-respondents will receive reminder email from the research centre for each of the follow-up surveys. All participants will receive a paper-based survey questionnaire from CRC at baseline and at each follow-up. Completed questionnaires will be returned to CRC in a sealed envelope.

\section{Primary outcomes}

Depression and anxiety

The Depression Anxiety and Stress Scales (DASS) is a widely used screening tool to assess symptoms of depression, anxiety and stress in community settings. ${ }^{52}$ The DASS comprises three subscales (ie, depression, anxiety and stress). The depression subscale measures dysphoria, hopelessness, devaluation of life, among others. The anxiety subscale measures autonomic arousal, skeletal musculature symptoms, situational anxiety, among others. The stress scale measures difficulty relaxation, nervous arousal, easily upset/agitated, among others. The short 21-item version (DASS 21, seven items in each of the three subscales) ${ }^{53}$ will be used in this study. Items are scored on a 4-point scale ranging from 0 (did not apply to me at all) to 3 (applied to me very much, or most of the time). In order to yield equivalent scores to the full version of DASS (42-item), the total score of each scale is multiplied by 2 and ranges from 0 to $42 .{ }^{53} \mathrm{~A}$ Vietnamese version of DASS 21 has been developed and tested, and its reliability and validity have been confirmed. ${ }^{54}$ The depression scale and the anxiety scale will be used to assess the depression and anxiety symptoms as primary outcomes in this study.

\section{Secondary outcomes}

Work engagement

Work engagement will be assessed using the short form of the Utrecht Work Engagement Scale (UWES). ${ }^{55}$ The UWES consists of three subscales (ie, vigour, dedication and absorption) comprising nine items. Items are scored on a 7-point scale ranging from 0 (never) to 6 (always). A total score is calculated from all nine items. The Vietnamese version will be developed and validated before the study.

\section{Sick leave days and self-reported work performance}

The WHO Health and Productivity Questionnaire is a self-report instrument designed to estimate the workplace costs of health problems in terms of self-reported sickness absence (absenteeism) and reduced job performance (presenteeism). ${ }^{56}$ Respondents will be asked to rate their overall work performance during the past 4 weeks. The item will be scored on an 11-point scale ranging from 0 (worst possible performance) to 10 (best possible performance). High scores indicate a high degree of perceived work performance. The Vietnamese version will be developed and validated before the study.

\section{Stress symptoms}

Stress symptoms will be assessed with the stress scales of DASS $21^{52-54}$ above.

\section{Psychosocial work environment}

The Job Content Questionnaire (JCQ) will be used to assess psychological job demands, control and support by coworkers and supervisors. ${ }^{57}$ The JCQ consists of a fiveitem psychological demand scale, a nine-item decision latitude scale, a four-item supervisor support scale and a 4-item coworker support scale. Items are scored on a 4-point Likert scale, ranging from 1 (strongly disagree) to 
4 (strongly agree). The Vietnamese version will be developed and validated before the study.

\section{Health-related quality of life}

Health-related QOL will be assessed with the EQ-5D-5L. ${ }^{58}$ The EQ-5D-5L consists of five items covering five dimensions (mobility, self-care, usual activities, pain/discomfort and anxiety/depression), each of which is rated as causing 'no problems' to 'unable to', and a visual analogue scale. It is a widely applied QOL instrument, and its reliability and validity are well established. ${ }^{58}$ The Vietnamese version has been developed and tested for its reliability and validity. ${ }^{59}$

\section{Improvement of knowledge and self-efficacy}

Respondents will be asked to rate their improvement of knowledge and self-efficacy regarding the two intervention programmes. Knowledge improvement will be assessed by asking participants, "How much knowledge do you have about...,' and self-efficacy improvement will be assessed by asking respondents 'How confident are you that you can do....' Both items are scored on a 5-point scale ranging from 0 (none) to 4 (enough). This scale is originally developed and has not yet been validated.

\section{Process evaluation}

\section{programme satisfaction and usage}

Participants in the intervention groups will be asked to rate their satisfaction with the intervention programme at the end of the intervention period. To evaluate any difference in adherence to the two intervention programmes, the usage of the intervention programmes will be collected from the records of the apps system. It is technically difficult to make the content of the two intervention programmes identical; the adaptation and modification process following the consultation with nurses in Vietnam make it more difficult. However, we still use the same CBT components (ie, behavioural activation, cognitive restructuring and problem solving) in both the programmes, keeping $50 \%$ of the content overlapping (see table 1 ).

\section{Contamination of information}

To evaluate contamination of information among participants, participants will be asked at follow-up survey: 'During the past 3 months, have you got to know information on stress management from your colleagues who used any smartphone-based stress management programmes?', with a response option, yes/no. This scale will be originally developed.

\section{Demographic characteristics}

Demographic data, such as age, gender, marital status, occupation, education, chronic disease and overtime hours during the past month will also be collected.

\section{Sample size calculation}

A required sample size was calculated for one of the outcome variables, that is, depressive symptoms assessed by DASS. Previous meta-analyses of web-based psychological intervention on improving workers' mental health in the workplace yielded effect sizes of 0.23 to $0.37 .{ }^{25}{ }^{26}$ To detect a small effect size (ie, 0.25 ) or more at an alpha error rate of 0.05 and a beta error rate of 0.15 , the estimated sample size was 289 participants in each group. With an anticipated dropout rate of $25 \%$, the necessary sample size was 361 participants per arm. The statistical power was calculated using the $\mathrm{G}^{*}$ Power 3 program. ${ }^{6061}$

\section{Randomisation}

Participants who fulfil the inclusion criteria will be randomly allocated to one of the three trial arms (two intervention groups or control group). Stratified permuted-block randomisation will be conducted as well. The block sizes of this study will be fixed to 3. Participants will be stratified into two strata according to the depression subscale score of DASS $(\geq 10$ or $<10)$ in the baseline survey. ${ }^{54}$ In addition to the analysis of the whole sample (to examine the universal intervention effect), we will also analyse data by a priori-defined subgroups (to examine the selective intervention effect). A stratified permuted block random table will be generated by an independent biostatistician. Enrolment will be conducted by a CRC, and assignment will be conducted by an independent research assistant. The stratified permuted-block random table will be password protected and blinded to the researcher. Only the research assistant will be able to access it during the work of random allocation.

\section{Statistical methods}

\section{Clinical efficacy}

For the main pooled analysis, a mixed model for repeated measures conditional growth model analysis with an unstructured covariance matrix will be conducted using a group (intervention and control) $\times$ time (baseline, 3-month and 7-month follow-ups) interaction as an indicator of intervention effect. For sensitivity analysis, a similar mixed model for repeated measures, but using the analysis of variance model, with an unstructured covariance matrix will be conducted. Missing values will be imputed applying the maximum likelihood estimation using the MIXED procedure. An intention-to-treat principle will be applied as well. The effect size indicators are twofold. We will estimate a regression coefficient for a group (each of the two intervention groups vs the control group) $\mathrm{x}$ time (baseline and two follow-ups) interaction using the MIXED procedure, which will be converted an effect size by dividing by a pooled SD at baseline and at follow-ups. Second, we will calculate Cohen's d among completers at baseline for each follow-up. The level of statistical significance for all analyses in this study will be set at 0.05 (two-tailed), and 95\% CIs will be calculated. For Cohen's d, the values of 0.2, 0.5 and 0.8 are generally interpreted as being suggestive of small, medium and large effects, respectively. ${ }^{62}$ For process measures, the $\chi^{2}$ test will be performed to examine the difference between the two intervention groups. All statistical analyses will be conducted using the SPSS Statistics V.22.0 (IBM). 


\section{Subgroup analysis}

The effectiveness of the programme may differ according to the initial severity of depressive symptoms. We will, therefore, use the stratification factor (ie, participants who scored $\geq 10$ in DASS depression subscale at the baseline survey) and analyse the results according to a priori-defined subgroups (selective intervention effect).

\section{Data monitoring}

A Data and Safety Monitoring Board (DSMB) will be set up, including an independent chair and at least two independent members. The DSMB will meet every 3 months after the first participant is randomised. The purpose of the meetings will be to review the report prepared by the CRC. The CRC will prepare DSMB reports to monitor recruitment progress and data collection (eg, percentage completing each follow-up).

\section{Patient and public involvement}

In the present study, the research question, the study design and the outcome measures were determined based on a discussion with representatives of hospital nurses in the target hospital (chief nurses). Senior nurses of the target hospital (who were not participants of the study) were invited to a meeting with researchers to review and comment on the intervention programmes based on their priorities, experience and preferences. The representatives of hospital nurses in the target hospital will help recruiting and conducting the study. The results of the study will be disseminated to all nurses in the hospital via a newsletter or with other media, after the study is done, with an opportunity for them to use the intervention programmes. In this RCT, the burden of the intervention will be assessed by participants themselves.

\section{ETHICS AND DISSEMINATION}

\section{Ethical and safety considerations}

Written informed consent will be obtained from all participants included in this study after full disclosure and explanation of the purpose and procedures of the study. Candidates will be informed that their participation is voluntary, that even after voluntarily participating they can withdraw from the study at any time without stating the reason and that neither participation nor withdrawal will cause any advantage or disadvantage to them.

We expect no adverse health effect from this intervention, except possibility for deterioration in depressive/ anxiety symptoms. We will provide the emergency phone call number and e-mail address at the central office. The CRC (TTran), who is a registered nurse, will then deal with the emergency call or e-mail first by herself, and then consult with the clinical supervisor (NK) to provide appropriate care.

\section{Data confidentiality}

Participants will complete a baseline/follow-up questionnaire with a sealed envelope and submit it to the research centre. After the survey, the collected data will be entered into a password-locked stand-alone PC by the CRC. The collected data will be stored as linkable anonymising data. The data will be accessible only by the CRC.

\section{Dissemination of research findings}

The main findings of this study will be disseminated via publications in peer-reviewed international journals. Presentations of study findings will also be offered at relevant research conferences, and local academic symposia and seminars. If the intervention programmes are found to produce a significant positive effect in this RCT, these programmes can be made available for all nurses in Vietnam in the future.

\section{Strengths and limitations}

The greatest strength of this study is its focus on the effect of the fully automated web-based smartphone application intervention programmes on improving depressive and anxiety symptoms among Vietnamese nurses using RCT design. This study is also intended to add evidence for the effect of e-stress management programmes on positive work outcomes (eg, work engagement and work performance) among nurses. To our knowledge, the present study will be the first to determine whether a fully automated smartphone-based stress management programme is effective in improving depressive and anxiety symptoms and relevant work outcomes among nurses in Vietnam, a middle-income country in Southeast Asia, using a well-designed study protocol. This study will contribute to future development of strategies in the primary prevention of depression and anxiety and promotion of positive mental health among nurses in the low-income and middle-income countries.

Another strength of this study is to contribute to the creation of a new option for mental health services if this RCT will demonstrate the effectiveness of e-stress management programmes developed in this project in a low-income and middle-income country context. Such programmes would provide hospital nurses in Vietnam with an opportunity to have access to a low-cost mental health service.

One of the major weaknesses of this study is that all outcomes will be measured by self-report, which may be affected by the perceptions of the participants or by situational factors at work. Next, participants will be recruited from full-time nurses of one big general hospital in Vietnam. Therefore, generalisation of the findings to nurses working under different contract and work environments may be limited. Third, a slight difference in the content between the two intervention programmes may also be a limitation in comparing the adherence between the free-choice programme and the fixed-order programme.

\section{Author affiliations}

${ }^{1}$ Department of Mental Health, Graduate School of Medicine, The University of Tokyo, Tokyo, Japan 
${ }^{2}$ Department of Occupational Health and Safety, Hanoi University of Public Health, Hanoi, Vietnam

${ }^{3}$ Faculty of Social Sciences - Behavior and Health Education, Hanoi University of Public Health, Hanoi, Vietnam

${ }^{4}$ Department of Psychiatric Nursing, Graduate School of Medicine, The University of Tokyo, Tokyo, Japan

${ }^{5}$ Bach Mai Hospital, Hanoi, Vietnam

${ }^{6}$ Nursing Office, Bach Mai Hospital, Hanoi, Vietnam

${ }^{7}$ Family Medicine \& Primary Care, Lee Kong Chian School of Medicine, Nanyang Technological University Singapore, Singapore

${ }^{8}$ Melbourne School of Population and Global Health, The University of Melbourne, Melbourne, Victoria, Australia

${ }^{9}$ Department of Public Health, Kitasato University School of Medicine, Sagamihara, Japan

Contributors KI, TTTT, HTN, KK, AS, TMB, AQN, QTN, KTN, GTHN, XTNT, TQT, MWBZ, HM, YS, NS, AT and NK conceived and designed the experiments. KI, TTTT, HTN, KK, AS, YS, NS and NK contributed reagents/materials/analysis tools. KI, TTTT, HTN, HM and NK wrote the paper. All authors read and approved the final paper.

Funding This research was supported by AMED under Grant Number JP17jk0110014 and JP18jk0110014.

Disclaimer The funder had no role in study design, data collection and analysis, decision to publish or preparation of the manuscript.

Competing interests NK reports grants from Infocom Corp, Fujitsu Ltd, Fujitsu Software Technologies and TAK Ltd, personal fees from Occupational Health Foundation, Japan Dental Association, Sekisui Chemicals, Junpukai Health Care Center, Osaka Chamber of Commerce and Industry, outside the submitted work.

Patient consent for publication Obtained.

Ethics approval The study procedures have been approved by the Research Ethics Review Board of Graduate School of Medicine/Faculty of Medicine, the University of Tokyo (no 11991) and the Ethical Review Board for Biomedical Research of Hanoi University of Public Health (no 346/2018/YTCC-HD3).

Provenance and peer review Not commissioned; externally peer reviewed.

Open access This is an open access article distributed in accordance with the Creative Commons Attribution Non Commercial (CC BY-NC 4.0) license, which permits others to distribute, remix, adapt, build upon this work non-commercially, and license their derivative works on different terms, provided the original work is properly cited, appropriate credit is given, any changes made indicated, and the use is non-commercial. See: http://creativecommons.org/licenses/by-nc/4.0/.

\section{REFERENCES}

1. McVicar A. Workplace stress in nursing: a literature review. J Adv Nurs 2003;44:633-42.

2. Ruotsalainen $\mathrm{JH}$, Verbeek $\mathrm{JH}$, Mariné $\mathrm{A}$, et al. Preventing occupational stress in healthcare workers. Cochrane Database Syst Rev 2015;4:CD002892.

3. Galdikien N, Asikainen P, Balčiūnas $\mathrm{S}$, et al. Do nurses feel stressed? A perspective from primary health care. Nurs Health Sci 2014;16:327-34.

4. de Boer J, Lok A, Van't Verlaat E, et al. Work-related critical incidents in hospital-based health care providers and the risk of post-traumatic stress symptoms, anxiety, and depression: a meta-analysis. Soc Sci Med 2011;73:316-26.

5. Gómez-Urquiza JL, Aneas-López AB, Fuente-Solana El, et al. Prevalence, Risk Factors, and Levels of Burnout Among Oncology Nurses: A Systematic Review. Oncol Nurs Forum 2016;43:E104-20.

6. Yassi A, Lockhart K. Work-relatedness of low back pain in nursing personnel: a systematic review. Int J Occup Environ Health 2013;19:223-44.

7. Trudel-Fitzgerald C, Chen Y, Singh A, et al. Psychiatric, Psychological, and Social Determinants of Health in the Nurses Health Study Cohorts. Am J Public Health 2016;106:1644-9.

8. Weinberg A, Creed F. Stress and psychiatric disorder in healthcare professionals and hospital staff. Lancet 2000;355:533-7.

9. Fleming KC, Evans JM, Chutka DS. Caregiver and clinician shortages in an aging nation. Mayo Clin Proc 2003;78:1026-40.

10. Duvall JJ, Andrews DR. Using a structured review of the literature to identify key factors associated with the current nursing shortage. $J$ Prof Nurs 2010;26:309-17.
11. Toh SG, Ang E, Devi MK. Systematic review on the relationship between the nursing shortage and job satisfaction, stress and burnout levels among nurses in oncology/haematology settings. Int $J$ Evid Based Healthc 2012;10:126-41.

12. Organization WH. The world health report 2006: working together for health: World Health Organization, 2006.

13. Elison NK, Verani AR, McCarthy C. National nursing and midwifery legislation in countries of South-East Asia with high HIV burdens. WHO South East Asia J Public Health 2015;4:12-19.

14. Nguyen HTT, Kitaoka K, Sukigara M, et al. Burnout Study of Clinical Nurses in Vietnam: Development of Job Burnout Model Based on Leiter and Maslach's Theory. Asian Nurs Res 2018;12:42-9.

15. Pongruengphant $R$, Tyson PD. When nurses cry: coping with occupational stress in Thailand. Int J Nurs Stud 2000;37:535-9.

16. Lwin PM, Rattanapan C, Laosee O. Nurses' intention to remain employed in hospitals: Understanding the shortage in the context of Myanmar. Int J Nurs Pract 2017;23:e12536.

17. Labrague LJ, McEnroe-Petitte DM, Gloe D, et al. Organizational politics, nurses' stress, burnout levels, turnover intention and job satisfaction. Int Nurs Rev 2017;64:109-16.

18. Chan KB, Lai G, Ko YC, Yc K, et al. Work stress among six professional groups: the Singapore experience. Soc Sci Med 2000;50:1415-32.

19. Salmasi S, Khan TM, Hong YH, et al. Medication Errors in the Southeast Asian Countries: A Systematic Review. PLoS One 2015;10:e0136545.

20. Joyce S, Modini M, Christensen H, et al. Workplace interventions for common mental disorders: a systematic meta-review. Psychol Med 2016;46:683-97.

21. Wan Mohd Yunus WMA, Musiat P, Brown JSL. Systematic review of universal and targeted workplace interventions for depression. Occup Environ Med 2018;75:66-75.

22. Richardson KM, Rothstein HR. Effects of occupational stress management intervention programs: a meta-analysis. J Occup Health Psychol 2008;13:69-93.

23. van der Klink JJ, Blonk RW, Schene AH, et al. The benefits of interventions for work-related stress. Am J Public Health 2001;91:270-6.

24. Ryan C, Bergin M, Chalder T, et al. Web-based interventions for the management of stress in the workplace: Focus, form, and efficacy. $J$ Occup Health 2017:59:215-36.

25. Carolan S, Harris PR, Cavanagh K. Improving Employee Well-Being and Effectiveness: Systematic Review and Meta-Analysis of WebBased Psychological Interventions Delivered in the Workplace. J Med Internet Res 2017;19:e271.

26. Stratton E, Lampit A, Choi I, et al. Effectiveness of eHealth interventions for reducing mental health conditions in employees: A systematic review and meta-analysis. PLoS One 2017;12:e0189904.

27. Hersch RK, Cook RF, Deitz DK, et al. Reducing nurses' stress: A randomized controlled trial of a web-based stress management program for nurses. Appl Nurs Res 2016;32:18-25.

28. Morrison LG, Yardley L, Powell J, et al. What design features are used in effective e-health interventions? A review using techniques from Critical Interpretive Synthesis. Telemed J E Health 2012;18:137-44.

29. Meyer B, Berger T, Caspar F, et al. Effectiveness of a novel integrative online treatment for depression (Deprexis): randomized controlled trial. J Med Internet Res 2009;11:e15.

30. Coulon SM, Monroe CM, West DS. A Systematic, Multi-domain Review of Mobile Smartphone Apps for Evidence-Based Stress Management. Am J Prev Med 2016;51:95-105.

31. Rathbone AL, Prescott J. The Use of Mobile Apps and SMS Messaging as Physical and Mental Health Interventions: Systematic Review. J Med Internet Res 2017;19:e295.

32. Chan AW, Tetzlaff JM, Gøtzsche PC, et al. SPIRIT 2013 explanation and elaboration: guidance for protocols of clinical trials. BMJ 2013;346:e7586.

33. Imamura K, Kawakami N, Furukawa TA, et al. Effects of an Internetbased cognitive behavioral therapy (iCBT) program in Manga format on improving subthreshold depressive symptoms among healthy workers: a randomized controlled trial. PLoS One 2014;9:e97167.

34. Sakuraya A, Imamura K, Kagami H, et al; Which e-learning stress management program improve psychological distress most?: a multi-arm randomized controlled trial. The 90th Annual Meeting of Japan Society for Occupational Health. Tokyo: Japan Society for Occupational Health, 2017:327.

35. Beck AT. Cognitive therapy of depression. New York: Guilford Press, 1979.

36. Lewinsohn PM. The Coping with depression course: a psychoeducational intervention for unipolar depression. Eugene, OR: Castalia Pub. Co., 1984. 
37. Lazarus RS, Folkman S. Coping and adaptation. The handbook of behavioral medicine, 1984:282-325.

38. Padesky CA, Greenberger D. Clinician's guide to Mind over mood. New York: Guilford Press, 1995.

39. Adams H. Further Clarifications of Case Formulation. Behaviour Therapist 1996;19:78.

40. Martell CR, Dimidjian S, Herman-Dunn R. Behavioral activation for depression: A clinician's guide: Guilford Press, 2013.

41. Ellis A. Humanistic psychotherapy: the rational-emotive approach. New York: Julian Press, 1973.

42. Ellis A, Harper RA. A new guide to rational living. Englewood Cliffs, N.J.: Prentice-Hall, 1975.

43. Bell AC, D'Zurilla TJ. Problem-solving therapy for depression: a meta-analysis. Clin Psychol Rev 2009;29:348-53.

44. D'Zurilla TJ, Goldfried MR. Problem solving and behavior modification. J Abnorm Psychol 1971;78:107-26.

45. Alberti RE. Differentiating Assertion and Aggression - Some Behavioral Guidelines - Reply. Behav Ther 1977;8:353-4.

46. Alberti RE, Emmons ML. Your perfect right: assertiveness and equality in your life and relationships. 8th ed. Atascadero, Calif: Impact Publishers, 2001.

47. Bower SA, Bower GH. Asserting yourself: a practical guide for positive change. Updated ed. Cambridge, MA: Da Capo Life Long, 2004.

48. Neff K. Self-compassion: an alternative conceptualization of a healthy attitude toward oneself. Self and Identity 2003;2:85-101.

49. Barnard LK, Curry JF. Self-compassion: Conceptualizations, correlates, \& interventions. Review of General Psychology 2011;15:289-303.

50. Wrzesniewski A, Dutton JE. Crafting a job: revisioning employees as active crafters of their work. Acad Manage Rev 2001;26:179-201.

51. Kessler RC, Andrews G, Colpe LJ, et al. Short screening scales to monitor population prevalences and trends in non-specific psychological distress. Psychol Med 2002;32:959-76.
52. Lovibond PF, Lovibond SH. The structure of negative emotional states: comparison of the Depression Anxiety Stress Scales (DASS) with the Beck Depression and Anxiety Inventories. Behav Res Ther 1995;33:335-43.

53. Henry JD, Crawford JR. The short-form version of the Depression Anxiety Stress Scales (DASS-21): construct validity and normative data in a large non-clinical sample. Br J Clin Psychol 2005;44(Pt 2):227-39.

54. Tran TD, Tran T, Fisher J. Validation of the depression anxiety stress scales (DASS) 21 as a screening instrument for depression and anxiety in a rural community-based cohort of northern Vietnamese women. BMC Psychiatry 2013;13:24

55. Schaufeli WB, Salanova M, González-romá V, et al. The measurement of engagement and burnout: A two sample confirmatory factor analytic approach. J Happiness Stud 2002;3:71-92.

56. Kessler RC, Barber C, Beck A, et al. The World Health Organization Health and Work Performance Questionnaire (HPQ). J Occup Environ Med 2003;45:156-74.

57. Karasek R, Brisson C, Kawakami N, et al. The Job Content Questionnaire (JCQ): an instrument for internationally comparative assessments of psychosocial job characteristics. J Occup Health Psychol 1998;3:322-55.

58. Herdman M, Gudex C, Lloyd A, et al. Development and preliminary testing of the new five-level version of EQ-5D (EQ-5D-5L). Qual Life Res 2011;20:1727-36.

59. Tran BX, Ohinmaa A, Nguyen LT, et al. Determinants of healthrelated quality of life in adults living with HIV in Vietnam. AIDS Care 2011;23:1236-45.

60. Faul F, Erdfelder E, Buchner A, et al. Statistical power analyses using G*Power 3.1: tests for correlation and regression analyses. Behav Res Methods 2009;41:1149-60.

61. Faul F, Erdfelder E, Lang AG, et al. G*Power 3: a flexible statistical power analysis program for the social, behavioral, and biomedical sciences. Behav Res Methods 2007;39:175-91.

62. Cohen J. A power primer. Psychol Bull 1992;112:155-9. 